\title{
Demiclosed principals and convergence theorems for asymptotically pseudocontractive nonself-mappings in intermediate sense
}

\author{
Yunpeng Zhang \\ College of Electric Power, North China University of Water Resources and Electric Power, Zhengzhou, 450011, China.
}

Communicated by $\mathrm{X}$. Qin

\begin{abstract}
In this work, we study fixed points of nonself-mappings which are asymptotically pseudocontractive in the intermediate sense via an implicit iterative process. Convergence analysis is investigated in the framework of Hilbert spaces. We also give strong convergence criteria for the class of mappings. (C)2017 All rights reserved.
\end{abstract}

Keywords: Asymptotically pseducontractive mapping, implicit algorithm, metric projection, convergence analysis. 2010 MSC: 47H09, 65J15.

\section{Introduction}

Fixed point theory of nonlinear operators provides us with a general and unified framework in which to study a wide class of problems arising in pure and applied sciences; see, for example, $[1,10,18,20,24]$ and the references therein. In addition to the existence results, many authors have extensively investigated the approximation of fixed points of nonlinear operators via various kind of iterative processes, in particular, the mean valued iterative process; see, $[2,3,6,7,19]$, and the references therein. In recent years, asymptotically nonexpansive mappings have been generalized and extended in several directions using novel and innovative techniques with a wide range of applications in pure and applied sciences based on iterative processes. From the manner of generating iterative sequences, there are two kinds of processes: explicit iterative processes and implicit iterative processes. Every iterative process has its advantage and disadvantage for fixed points of nonlinear operators. Under the case that both of them converge, we may prefer from the standpoint of computation the explicit one. It is known that explicit iterative processes fail to converge to fixed points of pseudocontractive mappings even that they are Lipschitz continuous, however, the implicit one does. In this paper, we study an implicit iterative process. Weak and strong convergence of the processes are obtained. We also give strong convergence criteria in the framework of Hilbert spaces.

\section{Preliminaries}

Let $\mathrm{H}$ be a real Hilbert space with the inner product $\langle\cdot, \cdot\rangle$ and the norm $\|\cdot\|$. Let $\mathrm{C}$ be a nonempty closed convex subset of $\mathrm{H}$ and let $\operatorname{Proj}_{\mathrm{C}}^{\mathrm{H}}$ be the metric projection from $\mathrm{H}$ onto $\mathrm{C}$.

Email address: zhangypliyl@yeah.net (Yunpeng Zhang)

doi:10.22436/jnsa.010.04.73 
Let $S: C \rightarrow C$ be a mapping. We denote Fix $(S)$ by the fixed point of the mapping $S$.

Recall that $S$ is said to be asymptotically nonexpansive iff there exists a sequence $\left\{k_{n}\right\} \subset[1, \infty)$ with $k_{n} \rightarrow 1$ as $n \rightarrow \infty$ such that

$$
\left\|S^{n} x-S^{n} y\right\| \leqslant k_{n}\|x-y\|, \quad \forall x, y \in C, n \geqslant 1 .
$$

$\mathrm{S}$ is said to be asymptotically nonexpansive in the intermediate sense iff it is continuous and the following inequality holds:

$$
\limsup _{n \rightarrow \infty} \sup _{x, y \in C}\left(\left\|S^{n} x-S^{n} y\right\|-\|x-y\|\right) \leqslant 0 .
$$

Putting

$$
\xi_{n}=\max \left\{0, \sup _{x, y \in C}\left(\left\|S^{n} x-S^{n} y\right\|-\|x-y\|\right)\right\},
$$

we see that $\xi_{n} \rightarrow 0$ as $n \rightarrow \infty$. Hence, we have the following

$$
\left\|S^{n} x-S^{n} y\right\| \leqslant\|x-y\|+\xi_{n}, \quad \forall x, y \in C, n \geqslant 1 .
$$

The class of asymptotically nonexpansive mappings in the intermediate sense was introduced by Kirk [12] (see also Bruck et al. [5]) as a generalization of the class of asymptotically nonexpansive mappings.

$S$ is said to be strictly pseudocontractive if there exists a constant $k \in[0,1)$ such that

$$
\|S x-S y\|^{2} \leqslant\|x-y\|^{2}+k\|(I-S) x-(I-S) y\|^{2}, \quad \forall x, y \in C .
$$

For such a case, $\mathrm{S}$ is also said to be a $\mathrm{k}$-strict pseudocontraction. The class of strict pseudocontractions was introduced by Browder and Petryshyn [4] in 1967. It is clear that every nonexpansive mapping is a 0 -strict pseudocontraction.

$S$ is said to be an asymptotically strict pseudocontraction if there exist a sequence $\left\{k_{n}\right\} \subset[1, \infty)$ with $k_{n} \rightarrow 1$ as $n \rightarrow \infty$ and a constant $k \in[0,1)$ such that

$$
\left\|S^{n} x-S^{n} y\right\|^{2} \leqslant k_{n}\|x-y\|^{2}+k\left\|\left(I-S^{n}\right) x-\left(I-S^{n}\right) y\right\|^{2}, \quad \forall x, y \in C, n \geqslant 1 .
$$

For such a case, $S$ is also said to be an asymptotically k-strict pseudocontraction. The class of asymptotically strict pseudocontractions is introduced by Liu [13] in 1996. It is clear that every asymptotically nonexpansive mapping is an asymptotical 0 -strict pseudocontraction.

$\mathrm{S}$ is said to be an asymptotically strict pseudocontraction in the intermediate sense if there exists a sequence $\left\{k_{n}\right\} \subset[1, \infty)$ with $k_{n} \rightarrow 1$ as $n \rightarrow \infty$ and a constant $k \in[0,1)$ such that

$$
\limsup _{n \rightarrow \infty} \sup _{x, y \in C}\left(\left\|S^{n} x-S^{n} y\right\|^{2}-k_{n}\|x-y\|^{2}-k\left\|\left(I-S^{n}\right) x-\left(I-S^{n}\right) y\right\|^{2}\right) \leqslant 0 .
$$

For such a case, $\mathrm{S}$ is also said to be an asymptotically $\mathrm{k}$-strict pseudocontraction in the intermediate sense. Putting

$$
\xi_{n}=\max \left\{0, \sup _{x, y \in C}\left(\left\|S^{n} x-S^{n} y\right\|^{2}-k_{n}\|x-y\|^{2}-k\left\|\left(I-S^{n}\right) x-\left(I-S^{n}\right) y\right\|^{2}\right)\right\},
$$

we see that $\xi_{n} \rightarrow 0$ as $n \rightarrow \infty$. Then we have the following:

$$
\left\|S^{n} x-S^{n} y\right\|^{2} \leqslant k_{n}\|x-y\|^{2}+k\left\|\left(I-S^{n}\right) x-\left(I-S^{n}\right) y\right\|^{2}+\xi_{n}, \quad \forall x, y \in C, n \geqslant 1 .
$$

The class of asymptotically strict pseudocontractions in the intermediate sense was introduced by Sahu et al. [22] as a generalization of the class of asymptotically strict pseudocontractions, see [22] for more details. We also remark that if $k_{n}=1$ for each $n \geqslant 1$ and $k=0$, then the class of asymptotically $k$ strict pseudocontractions in the intermediate sense is reduced to the class of asymptotically nonexpansive mappings in the intermediate sense. 
$S$ is said to be asymptotically pseudocontractive if there exists a sequence $\left\{k_{n}\right\} \subset[1, \infty)$ with $k_{n} \rightarrow 1$ as $n \rightarrow \infty$ such that

$$
\langle S x-S y, x-y\rangle \leqslant \frac{k_{n}+1}{2}\|x-y\|^{2}, \quad \forall x, y \in C .
$$

It is easy to see that it is equivalent to

$$
\left\|S^{n} x-S^{n} y\right\|^{2} \leqslant k_{n}\|x-y\|^{2}+\left\|\left(I-S^{n}\right) x-\left(I-S^{n}\right) y\right\|^{2}, \quad \forall x, y \in C, n \geqslant 1 .
$$

We remark that the class of asymptotically pseudocontractive mappings was introduced by Schu [23] in 1991. For an asymptotically pseudocontractive mapping S, Zhou [27] proved that if $S$ is also uniformly Lipschitz and uniformly asymptotically regular. Then $S$ has a nonempty fixed point set. Moreover Fix $(S)$ is closed and convex.

$S$ is said to be an asymptotically pseudocontractive mapping in the intermediate sense if there exists an sequence $\left\{k_{n}\right\} \subset[1, \infty)$ with $k_{n} \rightarrow 1$ as $n \rightarrow \infty$ such that

$$
\limsup _{n \rightarrow \infty} \sup _{x, y \in C}\left(\left\|S^{n} x-S^{n} y\right\|^{2}-k_{n}\|x-y\|^{2}-\left\|\left(I-S^{n}\right) x-\left(I-S^{n}\right) y\right\|^{2}\right) \leqslant 0 .
$$

It is easy to see that it is equivalent to

$$
\limsup _{n \rightarrow \infty} \sup _{x, y \in C}\left(\left\langle S^{n} x-S^{n} y, x-y\right\rangle-\frac{k_{n}+1}{2}\|x-y\|^{2}\right) \leqslant 0 .
$$

Put

$$
\xi_{n}=\max \left\{0, \sup _{x, y \in C}\left(\left\|S^{n} x-S^{n} y\right\|^{2}-k_{n}\|x-y\|^{2}-\left\|\left(I-S^{n}\right) x-\left(I-S^{n}\right) y\right\|^{2}\right)\right\} .
$$

Then we have the following

$$
\left\|S^{n} x-S^{n} y\right\|^{2} \leqslant k_{n}\|x-y\|^{2}+\left\|\left(I-S^{n}\right) x-\left(I-S^{n}\right) y\right\|^{2}+\xi_{n}, \quad \forall n \geqslant 1, \quad x, y \in C .
$$

It is easy to see that it is equivalent to

$$
\left\langle S^{n} x-S^{n} y, x-y\right\rangle \leqslant \frac{k_{n}+1}{2}\|x-y\|^{2}+\frac{\xi_{n}}{2}, \quad \forall n \geqslant 1, \quad x, y \in C .
$$

The class of asymptotically pseudocontractive mappings in the intermediate sense which includes the class of asymptotically pseudocontractive mappings and the class of asymptotically strict pseudocontractions in the intermediate sense as special cases.

In this paper, we consider the following nonself-mappings.

$\mathrm{T}: \mathrm{C} \rightarrow \mathrm{H}$ is said to be an asymptotically pseudocontractive mapping in the intermediate sense if there exists an sequence $\left\{k_{n}\right\} \subset[1, \infty)$ with $k_{n} \rightarrow 1$ as $n \rightarrow \infty$ such that

$\limsup _{n \rightarrow \infty} \sup _{x, y \in C}\left(\left\|\left(\operatorname{Proj}_{C}^{H} T\right)^{n} x-\left(\operatorname{Proj}_{C}^{H} T\right)^{n} y\right\|^{2}-k_{n}\|x-y\|^{2}-\left\|\left(I-\left(\operatorname{Proj}_{C}^{H} T\right)^{n}\right) x-\left(I-\left(\operatorname{Proj}_{C}^{H} T\right)^{n}\right) y\right\|^{2}\right) \leqslant 0$.

It is easy to see that it is equivalent to

$$
\limsup _{n \rightarrow \infty} \sup _{x, y \in C}\left(\left\langle\left(\operatorname{Proj}_{C}^{\mathrm{H}} \mathrm{T}\right)^{\mathrm{n}} x-\left(\operatorname{Proj}_{C}^{\mathrm{H}} \mathrm{T}\right)^{\mathrm{n}} y, x-y\right\rangle-\frac{k_{n}+1}{2}\|x-y\|^{2}\right) \leqslant 0 .
$$

Put

$$
\begin{gathered}
\xi_{n}=\max \left\{0, \sup _{x, y \in C}\left(\left\|\left(\operatorname{Proj}_{C}^{\mathrm{H}} \mathrm{T}\right)^{n} x-\left(\operatorname{Proj}_{C}^{\mathrm{H}} \mathrm{T}\right)^{n} y\right\|^{2}-k_{n}\|x-y\|^{2}\right.\right. \\
\left.\left.-\left\|\left(I-\left(\operatorname{Proj}_{C}^{\mathrm{H}} \mathrm{T}\right)^{\mathrm{n}}\right) x-\left(I-\left(\operatorname{Proj}_{\mathrm{C}}^{\mathrm{H}} \mathrm{T}\right)^{n}\right) y\right\|^{2}\right)\right\} .
\end{gathered}
$$


Then we have the following

$$
\begin{aligned}
\left\|\left(\operatorname{Proj}_{C}^{\mathrm{H}} T\right)^{n} x-\left(\operatorname{Proj}_{C}^{\mathrm{H}} T\right)^{n} y\right\|^{2} \leqslant & k_{n}\|x-y\|^{2}+\|\left(I-\left(\operatorname{Proj}_{C}^{\mathrm{H}} T\right)^{n}\right) x \\
& -\left(I-\left(\operatorname{Proj}_{C}^{\mathrm{H}} T\right)^{n}\right) y \|^{2}+\xi_{n}, \quad \forall n \geqslant 1, \quad x, y \in C .
\end{aligned}
$$

It is easy to see that it is equivalent to

$$
\left\langle\left(\operatorname{Proj}_{C}^{\mathrm{H}} \mathrm{T}\right)^{\mathrm{n}} x-\left(\operatorname{Proj}_{C}^{\mathrm{H}} \mathrm{T}\right)^{\mathrm{n}} y, x-y\right\rangle \leqslant \frac{k_{n}+1}{2}\|x-y\|^{2}+\frac{\xi_{n}}{2}, \quad \forall n \geqslant 1, \quad x, y \in C .
$$

Implicit iterative algorithms, which are complementary algorithms to the explicit iterative algorithms, have been extensively investigated for fixed points of asymptotically nonexpansive mappings and their extensions; see $[8,11,16,17]$, and the references therein. In this paper, we study the class of asymptotically pseudocontractive nonself-mapping in the intermediate sense based on a one step implicit iterative algorithm. We obtain some convergence theorems of common fixed points in the framework of Hilbert spaces. The results presented in this paper mainly improve the corresponding results in Khan et al. [9], Kim et al. [11], Lv [14], Wang [26] and Zhou [27].

In order to prove our main results, we need the following tools.

Recall that a space $X$ is said to satisfy Opial's condition [15], if for each sequence $\left\{x_{n}\right\}$ in $X$, the convergence $x_{n} \rightarrow x$ weakly implies that

$$
\liminf _{n \rightarrow \infty}\left\|x_{n}-x\right\|<\liminf _{n \rightarrow \infty}\left\|x_{n}-y\right\|, \quad \forall y \in E(y \neq x)
$$

Recall that a mapping $\mathrm{T}: \mathrm{C} \rightarrow \mathrm{H}$ is semicompact if any sequence $\left\{x_{n}\right\}$ in $\mathrm{C}$ satisfying

$$
\lim _{n \rightarrow \infty}\left\|x_{n}-\left(\operatorname{Proj}_{C}^{H} T\right) x_{n}\right\|=0,
$$

has a convergent subsequence.

Lemma 2.1 ([25]). Let $\left\{a_{n}\right\},\left\{b_{n}\right\}$ and $\left\{c_{n}\right\}$ be three nonnegative sequences satisfying the following condition:

$$
a_{n+1} \leqslant\left(1+b_{n}\right) a_{n}+c_{n}, \quad \forall n \geqslant n_{0},
$$

where $\mathrm{n}_{0}$ is some nonnegative integer, $\sum_{n=1}^{\infty} b_{n}<\infty$ and $\sum_{n=1}^{\infty} c_{n}<\infty$. Then the limit $\lim _{n \rightarrow \infty} a_{n}$ exists.

Lemma 2.2 ([21]). In a real Hilbert space, the following inequality holds

$$
\|a x+(1-a) y\|^{2}=a\|x\|^{2}+(1-a)\|y\|^{2}-a(1-a)\|x-y\|^{2}, \quad \forall a \in[0,1], \quad x, y \in H .
$$

\section{Demiclosed principals}

Theorem 3.1. Let $\mathrm{C}$ be a nonempty closed convex bounded subset of a Hilbert space $\mathrm{H}$. Let Proj ${ }_{\mathrm{C}}^{\mathrm{H}}$ be the metric projection from $\mathrm{H}$ onto $\mathrm{C}$. Let $\mathrm{T}: \mathrm{C} \rightarrow \mathrm{E}$ be a uniformly L-Lipschitz continuous and asymptotically pseudocontractive mapping in the intermediate sense. Let $\left\{x_{n}\right\}$ be a sequence in $C$ with $x_{n} \rightarrow x$ and $\lim _{n \rightarrow \infty} \| x_{n}-$ Proj ${ }_{C}^{H} T x_{n} \|=0$. Then $\mathrm{x}$ is a fixed point of $\mathrm{T}$.

Proof. Choose $k \in\left(0, \frac{1}{\mathrm{~L}+1}\right)$ and define $y_{m, k}=\kappa\left(\operatorname{Proj}_{C}^{\mathrm{H}} \mathrm{T}\right)^{\mathrm{m}} x+(1-\kappa) x$ for arbitrary but fixed $m \geqslant 1$. Notice that

$$
\begin{aligned}
\left\|\left(\operatorname{Proj}_{C}^{\mathrm{H}} \mathrm{T}\right)^{m^{2}} x_{n}-x_{n}\right\| \leqslant & \left\|x_{n}-\left(\operatorname{Proj}_{C}^{\mathrm{H}} \mathrm{T}\right) x_{n}\right\|+\left\|\left(\operatorname{Proj}_{C}^{\mathrm{H}} \mathrm{T}\right) x_{n}-\left(\operatorname{Proj}_{C}^{\mathrm{H}} \mathrm{T}\right)^{2} x_{n}\right\|+\cdots \\
& +\left\|\left(\operatorname{Proj}_{C}^{\mathrm{H}} \mathrm{T}\right)^{m-1} x_{n}-\left(\operatorname{Proj}_{C}^{\mathrm{H}} \mathrm{T}\right)^{m_{1}} x_{n}\right\| \\
\leqslant & (\mathrm{L}(m-1)+1)\left\|x_{n}-\left(\operatorname{Proj}_{C}^{\mathrm{H}} \mathrm{T}\right) x_{n}\right\| .
\end{aligned}
$$


This implies that

$$
\lim _{n \rightarrow \infty}\left\|\left(\operatorname{Proj}_{C}^{H} \mathrm{~T}\right)^{m} \chi_{n}-x_{n}\right\|=0
$$

On the other hand, we have

$$
\begin{aligned}
\left\langle\bar{x}-y_{m, k}, y_{m, k}-\left(\operatorname{Proj}_{C}^{H} T\right)^{m} y_{m, k}\right\rangle & \\
= & \left\langle\bar{x}-x_{n}, y_{m, k}-\left(\operatorname{Proj}_{C}^{H} T\right)^{m} y_{m, k}\right\rangle+\left\langle x_{n}-y_{m, k}, y_{m, k}-\left(\operatorname{Proj}_{C}^{H} T\right)^{m} y_{m, k}\right\rangle \\
= & \left\langle\bar{x}-x_{n}, y_{m, k}-\left(\operatorname{Proj}_{C}^{H} T\right)^{m} y_{m, k}\right\rangle+\left\langle x_{n}-y_{m, k}\left(\operatorname{Proj}_{C}^{H} T\right)^{m} x_{n}-\left(\operatorname{Proj}_{C}^{H} T\right)^{m} y_{m, k}\right\rangle \\
& -\left\langle x_{n}-y_{m, \alpha}, x_{n}-y_{m, \alpha}\right\rangle+\left\langle x_{n}-y_{m, k}, x_{n}-\left(\operatorname{Proj}_{C}^{H} T\right)^{m} x_{n}\right\rangle \\
\leqslant & \left\langle\bar{x}-x_{n}, y_{m, k}-\left(\operatorname{Proj}_{C}^{H} T\right)^{m} y_{m, k}\right\rangle+\frac{k_{m}-1}{2}\left\|x_{n}-y_{m, k}\right\|^{2}+\frac{\xi_{m}}{2} \\
& +\left\|x_{n}-y_{m, k}\right\|\left\|x_{n}-\left(\operatorname{Proj}_{C}^{H} T\right)^{m} x_{n}\right\| .
\end{aligned}
$$

From (3.1), one has

$$
\left\langle\bar{x}-y_{m, k}, y_{m, k}-\left(\operatorname{Proj}_{C}^{H} T\right)^{m} y_{m, k}\right\rangle \leqslant \frac{k_{m}-1}{2}\left\|x_{n}-y_{m, k}\right\|^{2}+\frac{\xi_{m}}{2} .
$$

Note that

$$
\begin{aligned}
\left\langle\bar{x}-y_{m, k}\left(\bar{x}-\left(\operatorname{Proj}_{C}^{H} T\right)^{m} \bar{x}\right)-\left(y_{m, k}-\left(\operatorname{Proj}_{C}^{H} T\right)^{m} y_{m, k}\right)\right\rangle & \leqslant(1+L)\left\|\bar{x}-y_{m, k}\right\|^{2} \\
& =k^{2}(1+L)\left\|\bar{x}-\left(\operatorname{Proj}_{C}^{H} T\right)^{m} \bar{x}\right\|^{2},
\end{aligned}
$$

and

$$
\begin{aligned}
& \left\|\bar{x}-\left(\operatorname{Proj}_{C}^{\mathrm{H}} \mathrm{T}\right)^{\mathrm{m}} \overline{\mathrm{x}}\right\|^{2}=\left\langle\bar{\chi}-\left(\operatorname{Proj}_{\mathrm{C}}^{\mathrm{H}} \mathrm{T}\right)^{\mathrm{m}} \overline{\mathrm{x}}, \overline{\mathrm{x}}-\left(\operatorname{Proj}_{\mathrm{C}}^{\mathrm{H}} \mathrm{T}\right)^{\mathrm{m}} \overline{\mathrm{x}}\right\rangle \\
& =\frac{1}{\kappa}\left\langle\bar{x}-y_{m, k}, \bar{x}-\left(\operatorname{Proj}_{C}^{H} T\right)^{m} \bar{x}\right\rangle \\
& =\frac{1}{\kappa}\left\langle\bar{x}-y_{m, k}\left(\bar{x}-\left(\operatorname{Proj}_{C}^{H} T\right)^{m} \bar{x}\right)-\left(y_{m, k}-\left(\operatorname{Proj}_{C}^{H} T\right)^{m} y_{m, k}\right)\right\rangle \\
& +\frac{1}{k}\left\langle\bar{x}-y_{m, k}, y_{m, k}-\left(\operatorname{Proj}_{C}^{H} T\right)^{m} y_{m, k}\right\rangle \text {. }
\end{aligned}
$$

Substituting (3.2) and (3.3) into (3.4), we arrive at

$$
(1-(1+\mathrm{L}) \kappa)\left\|\bar{x}-\left(\operatorname{Proj}_{C}^{\mathrm{H}} \mathrm{T}\right)^{\mathrm{m}} \overline{\mathrm{x}}\right\|^{2} \leqslant \frac{k_{\mathrm{m}}-1}{2}\left\|x_{n}-y_{m, k}\right\|^{2}+\frac{\xi_{m}}{2} .
$$

Letting $m \rightarrow \infty$ in (3.5), we see that $\left(\operatorname{Proj}_{C}^{H} T\right){ }^{m} \bar{x} \rightarrow \bar{x}$. Since (Proj ${ }_{C}^{H} T$ ) is uniformly L-Lipschitz, we can obtain that $\bar{x}=\left(\operatorname{Proj}_{C}^{H} T\right) \bar{x}$. This means that $\bar{x} \in \operatorname{Fix}\left(\operatorname{Proj}_{C}^{\mathrm{H}} T\right)=\operatorname{Fix}(T)$. This completes the proof.

\section{Weak convergence theorems}

Theorem 4.1. Let $\mathrm{C}$ be a nonempty closed convex subset of a Hilbert space $\mathrm{H}$ and let $\mathrm{Proj}_{\mathrm{C}}^{\mathrm{H}}$ be the metric projection from $\mathrm{H}$ onto $\mathrm{C}$. Let $\mathrm{T}_{i}: \mathrm{C} \rightarrow \mathrm{E}$ be a uniformly $\mathrm{L}_{i}$-Lipschitz continuous and asymptotically pseudocontractive mapping in the intermediate sense with the sequence $\left\{k_{n, i}\right\} \subset[1, \infty)$ such that $\sum_{n=1}^{\infty}\left(k_{n, i}-1\right)<\infty$ for each $1 \leqslant i \leqslant N$, where $N \geqslant 1$ is some positive integer. Let

$$
\xi_{(n, i)}=\max \left\{0, \sup _{x, y \in C}\left(\left\|T_{i}^{n} x-T_{i}^{n} y\right\|^{2}-k_{(n, i)}\|x-y\|^{2}-\left\|\left(I-T_{i}^{n}\right) x-\left(I-T_{i}^{n}\right) y\right\|^{2}\right)\right\},
$$

for each $1 \leqslant i \leqslant N$. Assume that the common fixed point set $\cap_{i=1}^{N}$ Fix $\left(T_{i}\right)$ is nonempty. Let $\left\{x_{n}\right\}_{\mathfrak{n}=0}^{\infty}$ be a sequence generated in the following manner:

$$
x_{n}=\alpha_{n} x_{n-1}+\left(1-\alpha_{n}\right)\left(\operatorname{Proj}_{C}^{H} T_{i(n)}\right)^{h(n)} x_{n}, \quad \forall n \geqslant 1 .
$$

Assume that the control sequence $\left\{\alpha_{n}\right\}$ in $(0,1)$ satisfies the following restrictions $0<1-\frac{1}{\mathrm{~L}}<\mathrm{a} \leqslant \alpha_{n} \leqslant \mathrm{~b}<1$, where $L=\max \left\{L_{i}: 1 \leqslant i \leqslant N\right\}, \forall n \geqslant 1, \sum_{n=1}^{\infty} \xi_{n}<\infty$, where $\xi_{n}=\max \left\{\xi_{(n, i)}: 1 \leqslant i \leqslant N\right\}$. Then $\left\{x_{n}\right\}$ converges weakly to some point in $\cap_{i=1}^{\mathrm{N}} \operatorname{Fix}\left(\mathrm{T}_{i}\right)$. 
Proof. The proof is split into four steps.

Step 1. Show that $\left\{x_{n}\right\}$ is well-defined.

Define mappings $C_{n}: C \rightarrow C$ by

$$
C_{n}(x)=\left(1-\alpha_{n}\right)\left(\operatorname{Proj}_{C}^{H} T_{i(n)}\right)^{h(n)} x+\alpha_{n} x_{n-1}, \quad \forall x \in C, \quad n \geqslant 1 .
$$

Since each $T_{i}$ is Lipschitz continuous, we have

$$
\begin{aligned}
\left\|C_{n}(x)-C_{n}(y)\right\|= & \|\left(\alpha_{n} x_{n-1}+\left(1-\alpha_{n}\right)\left(\operatorname{Proj}_{C}^{H} T_{i(n)}\right)^{h(n)} x\right) \\
& -\left(\alpha_{n} x_{n-1}+\left(1-\alpha_{n}\right)\left(\operatorname{Proj}_{C}^{H} T_{i(n)}\right)^{h(n)} y\right) \| \\
\leqslant & \left(1-\alpha_{n}\right) L\|x-y\| \\
\leqslant & L(1-a)\|x-y\|, \quad \forall x, y \in C .
\end{aligned}
$$

This shows that $C_{n}$ is a contraction for each $n \geqslant 1$. By the Banach contraction principle, we see that there exists a unique fixed point $x_{n} \in C$ such that

$$
x_{n}=\left(1-\alpha_{n}\right)\left(\operatorname{Proj}_{C}^{H} T_{i(n)}\right)^{h(n)} x_{n}+\alpha_{n} x_{n-1}, \quad \forall n \geqslant 1 .
$$

This yields that $\left\{x_{n}\right\}$ is well-defined. This completes the first step.

Step 2. Show that $\left\{x_{n}\right\}$ is bounded.

Letting $k_{n}=\max \left\{k_{n, i}: 1 \leqslant i \leqslant N\right\}$ and fixing $p \in \cap_{i=1}^{N} \operatorname{Fix}\left(T_{i}\right)$, we see from Lemma 2.2 that

$$
\begin{aligned}
\left\|x_{n}-p\right\|^{2}= & \left(1-\alpha_{n}\right)\left\|\left(\operatorname{Proj}_{C}^{H} T_{i(n)}\right)^{h(n)} x_{n}-p\right\|^{2}-\alpha_{n}\left(1-\alpha_{n}\right)\left\|\left(\operatorname{Proj}_{C}^{H} T_{i(n)}\right)^{h(n)} x_{n}-x_{n-1}\right\|^{2} \\
& +\alpha_{n}\left\|x_{n-1}-p\right\|^{2} \\
\leqslant & \left(1-\alpha_{n}\right)\left(k_{h(n)}\left\|x_{n}-p\right\|^{2}+\left\|\left(\operatorname{Proj}_{C}^{H} T_{i(n)}\right)^{h(n)} x_{n}-x_{n}\right\|^{2}+\xi_{n}\right)+\alpha_{n}\left\|x_{n-1}-p\right\|^{2} \\
& -\alpha_{n}\left(1-\alpha_{n}\right)\left\|\left(\operatorname{Proj}_{C}^{H} T_{i(n)}\right)^{h(n)} x_{n}-x_{n-1}\right\|^{2} \\
\leqslant & \left(1-\alpha_{n}\right) k_{h(n)}\left\|x_{n}-p\right\|^{2}+\left(1-\alpha_{n}\right)\left\|\left(\operatorname{Proj}_{C}^{H} T_{i(n)}\right)^{h(n)} x_{n}-x_{n}\right\|^{2}+\alpha_{n}\left\|x_{n-1}-p\right\|^{2} \\
& -\alpha_{n}\left(1-\alpha_{n}\right)\left\|\left(\operatorname{Proj}_{C}^{H} T_{i(n)}\right)^{h(n)} x_{n}-x_{n-1}\right\|^{2}+\xi_{n} \\
\leqslant & \alpha_{n}\left\|x_{n-1}-p\right\|^{2}+\left(1-\alpha_{n}\right) k_{h(n)}\left\|x_{n}-p\right\|^{2}-\left(1-\alpha_{n}\right)^{2} \alpha_{n}\left\|\left(\operatorname{Proj}_{C}^{H} T_{i(n)}\right)^{h(n)} x_{n}-x_{n-1}\right\|^{2}+\xi_{n} .
\end{aligned}
$$

From the restriction imposed on $\left\{\alpha_{n}\right\}$, we see that there exists some $n_{0}$ such that

$$
\left(1-\alpha_{\mathfrak{n}}\right) k_{\mathrm{h}(\mathrm{n})}<\left(1+\frac{\mathrm{a}}{2(1-\mathrm{a})}\right)(1-\mathrm{a})<1, \quad \forall \mathrm{n} \geqslant \mathrm{n}_{0} .
$$

Hence, we have

$$
\left\|x_{n}-p\right\|^{2} \leqslant\left(1+\frac{k_{h(n)}-1}{1-M}\right)\left\|x_{n-1}-p\right\|^{2}+\frac{\xi_{n}}{1-M^{\prime}}, \forall n \geqslant n_{0},
$$

where $M=\left(1+\frac{a}{2(1-a)}\right)(1-a)$. Using Lemma 2.1, we find that $\lim _{n \rightarrow \infty}\left\|x_{n}-p\right\|$ exists. Hence, $\left\{x_{n}\right\}$ is bounded. This completes the second step.

Step 3. Show that

$$
\lim _{n \rightarrow \infty}\left\|\left(\operatorname{Proj}_{C}^{H} T_{r}\right) x_{n}-x_{n}\right\|=0, \quad \forall r \in\{1,2, \cdots, N\}
$$

From (4.1), we have

$$
\left(1-\alpha_{n}\right)^{2} \alpha_{n}\left\|x_{n-1}-\left(\operatorname{Proj}_{C}^{H} T_{i(n)}\right)^{h(n)} x_{n}\right\|^{2} \leqslant\left(k_{h(n)}-1\right)\left\|x_{n}-p\right\|^{2}+\alpha_{n}\left(\left\|x_{n-1}-p\right\|^{2}-\left\|x_{n}-p\right\|^{2}\right)+\xi_{n} .
$$

This implies that

$$
\lim _{n \rightarrow \infty}\left\|\left(\operatorname{Proj}_{C}^{H} T_{i(n)}\right)^{h(n)} x_{n}-x_{n-1}\right\|=0
$$


Using (4.2) and the following fact

$$
\left\|x_{n}-x_{n-1}\right\| \leqslant\left\|\left(\operatorname{Proj}_{C}^{H} T_{i(n)}\right)^{h(n)} x_{n}-x_{n-1}\right\|,
$$

we have

$$
\lim _{n \rightarrow \infty}\left\|x_{n}-x_{n-1}\right\|=0
$$

On the other hand, we have

$$
\begin{aligned}
\|\left(\operatorname{Proj}_{C}^{H} T_{i(n)}\right)^{h(n)} & x_{n-1}-x_{n-1} \| \\
& \leqslant\left\|\left(\operatorname{Proj}_{C}^{H} T_{i(n)}\right)^{h(n)} x_{n}-\left(\operatorname{Proj}_{C}^{H} T_{i(n)}\right)^{h(n)} x_{n-1}\right\|+\left\|x_{n-1}-\left(\operatorname{Proj}_{C}^{H} T_{i(n)}\right)^{h(n)} x_{n}\right\| \\
& \leqslant L\left\|x_{n}-x_{n-1}\right\|+\left\|x_{n-1}-\left(\operatorname{Proj}_{C}^{H} T_{i(n)}\right)^{h(n)} x_{n}\right\| .
\end{aligned}
$$

It follows from (4.2) and (4.3) that

$$
\lim _{n \rightarrow \infty}\left\|x_{n-1}-T_{i(n)}^{h(n)} x_{n-1}\right\|=0 .
$$

Since for any positive integer $n>N$, it can be written as $n=(h(n)-1) N+i(n)$, where $i(n) \in\{1,2, \cdots, N\}$. Hence, we have

$$
\begin{aligned}
\| x_{n-1}- & \left(\operatorname{Proj}_{C}^{H} T_{n}\right) x_{n-1} \| \\
\leqslant & \left\|\left(\operatorname{Proj}_{C}^{H} T_{i(n)}\right)^{h(n)} x_{n-1}-\left(\operatorname{Proj}_{C}^{H} T_{n}\right) x_{n-1}\right\|+\left\|x_{n-1}-\left(\operatorname{Proj}_{C}^{H} T_{i(n)}\right)^{h(n)} x_{n-1}\right\| \\
\leqslant & \left\|x_{n-1}-\left(\operatorname{Proj}_{C}^{H} T_{i(n)}\right)^{h(n)} x_{n-1}\right\|+L\left\|\left(\operatorname{Proj}_{C}^{H} T_{i(n)}\right)^{h(n)-1} x_{n-1}-x_{n-1}\right\| \\
\leqslant & \left\|x_{n-1}-\left(\operatorname{Proj}_{C}^{H} T_{i(n)}\right)^{h(n)} x_{n-1}\right\| \\
& +L\left(\left\|\left(\operatorname{Proj}_{C}^{H} T_{i(n)}\right)^{h(n)-1} x_{n-1}-\left(\operatorname{Proj}_{C}^{H} T_{i(n-N)}\right)^{h(n)-1} x_{n-N}\right\|\right. \\
& \left.+\left\|\left(\operatorname{Proj}_{C}^{H} T_{i(n-N)}\right)^{h(n)-1} x_{n-N}-x_{(n-N)-1}\right\|+\left\|x_{(n-N)-1}-x_{n-1}\right\|\right) .
\end{aligned}
$$

Since for each $n>N, n=(n-N)(\bmod N)$, one finds that

$$
n-N=((h(n)-1)-1) N+i(n)=(h(n-N)-1) N+i(n-N) \text {. }
$$

That is,

$$
i(n-N)=i(n), \quad h(n-N)=h(n)-1 .
$$

Since each $T_{r}$ is Lipschitz, we have

$$
\left\|\left(\operatorname{Proj}_{C}^{H} T_{i(n-N)}\right)^{h(n)-1} x_{n-N}-x_{(n-N)-1}\right\|=\left\|\left(\operatorname{Proj}_{C}^{H} T_{i(n-N)}\right)^{h(n-N)} x_{n-N}-x_{(n-N)-1}\right\|,
$$

and

$$
\begin{aligned}
\|\left(\operatorname{Proj}_{C}^{H} T_{i(n)}\right)^{h(n)-1} x_{n-1} & -\left(\operatorname{Proj}_{C}^{H} T_{i(n-N)}\right)^{h(n)-1} x_{n-N} \| \\
& =\left\|\left(\operatorname{Proj}_{C}^{H} T_{i(n)}\right)^{h(n)-1} x_{n-1}-\left(\operatorname{Proj}_{C}^{H} T_{i(n)}\right)^{h(n)-1} x_{n-N}\right\| \\
& \leqslant L\left\|x_{n-N}-x_{n-1}\right\| .
\end{aligned}
$$

Using (4.5), (4.6) and (4.7), one has

$$
\begin{aligned}
\left\|\left(\operatorname{Proj}_{C}^{H} T_{n}\right) x_{n-1}-x_{n-1}\right\| \leqslant & \left\|\left(\operatorname{Proj}_{C}^{H} T_{i(n)}\right)^{h(n)} x_{n-1}-x_{n-1}\right\|+L\left(L\left\|x_{n-N}-x_{n-1}\right\|\right. \\
& \left.+\left\|\left(\operatorname{Proj}_{C}^{H} T_{i(n-N)}\right)^{h(n-N)} x_{n-N}-x_{(n-N)-1}\right\|+\left\|x_{n-1}-x_{(n-N)-1}\right\|\right) .
\end{aligned}
$$

It follows from (4.2), (4.3) and (4.4) that

$$
\lim _{n \rightarrow \infty}\left\|\left(\operatorname{Proj}_{C}^{H} T_{n}\right) x_{n-1}-x_{n-1}\right\|=0
$$

This further implies that

$$
\lim _{n \rightarrow \infty}\left\|x_{n}-\left(\operatorname{Proj}_{C}^{H} T_{n}\right) x_{n}\right\|=0
$$


On the other hand, one has

$$
\begin{aligned}
\| x_{n}- & \left(\operatorname{Proj}_{C}^{H} T_{n+m}\right) x_{n} \| \\
& \leqslant\left\|x_{n+m}-\left(\operatorname{Proj}_{C}^{H} T_{n+m}\right) x_{n+m}\right\|+\left\|\left(\operatorname{Proj}_{C}^{H} T_{n+m}\right) x_{n+m}-\left(\operatorname{Proj}_{C}^{H} T_{n+m}\right) x_{n}\right\|+\left\|x_{n}-x_{n+m}\right\| \\
& \leqslant\left\|x_{n+m}-\left(\operatorname{Proj}_{C}^{H} T_{n+m}\right) x_{n+m}\right\|+(1+L)\left\|x_{n}-x_{n+m}\right\| .
\end{aligned}
$$

Using (4.3) and (4.8), one sees that $\lim _{n \rightarrow \infty}\left\|x_{n}-\left(\operatorname{Proj}_{C}^{H} T_{n+m}\right) x_{n}\right\|=0$ for each $1 \leqslant m \leqslant N$. Therefore, we have

$$
\lim _{n \rightarrow \infty}\left\|x_{n}-\left(\operatorname{Proj}_{C}^{H} \mathrm{~T}_{r}\right) x_{n}\right\|=0, \quad \forall r \in\{1,2, \ldots, N\}
$$

This completes the third step.

Step 4. Show that $x_{n} \rightarrow \bar{x} \in \cap_{i=1}^{N} F i x\left(T_{i}\right)$.

Since $\left\{x_{n}\right\}$ is a bounded sequence, we see that there exists a subsequence $\left\{x_{n_{i}}\right\} \subset\left\{x_{n}\right\}$ such that $\left\{x_{n_{i}}\right\}$ converges weakly to a point $\bar{x}$. From Theorem 3.1, we obtain that $\bar{x} \in \cap_{i=1}^{N} \operatorname{Fix}\left(T_{i}\right)$. Let $\left\{x_{n_{j}}\right\}$ be another subsequence of $\left\{x_{n}\right\}$ such that $\left\{x_{n_{j}}\right\}$ converges weakly to $x^{*} \in C$, where $x^{*} \neq \bar{x}$. From the above argument, we have $x^{*} \in \cap_{i=1}^{N} \operatorname{Fix}\left(T_{i}\right)$. Since $\lim _{n \rightarrow \infty}\left\|x_{n}-x\right\|$ exists for all $x \in \cap_{i=1}^{N} F i x\left(T_{i}\right)$. Without loss of generality, we assume $\lim _{n \rightarrow \infty}\left\|x_{n}-\bar{x}\right\|=d>0$, where $d$ is a nonnegative number. By virtue of the Opial property of space $\mathrm{H}$, we see that

$$
\begin{aligned}
d & =\liminf _{n \rightarrow \infty}\left\|x_{n}-\bar{x}\right\|=\liminf _{i \rightarrow \infty}\left\|x_{n_{i}}-\bar{x}\right\|<\liminf _{i \rightarrow \infty}\left\|x_{n_{i}}-x^{*}\right\| \\
& =\liminf _{j \rightarrow \infty}\left\|x_{n_{j}}-x^{*}\right\|<\liminf _{j \rightarrow \infty}\left\|x_{n_{j}}-\bar{x}\right\|=\liminf _{n \rightarrow \infty}\left\|x_{n}-\bar{x}\right\|=d .
\end{aligned}
$$

This is a contradiction. Hence $\bar{x}=x^{*}$. This shows that $\left\{x_{n}\right\}$ converges weakly to $\bar{x} \in \cap_{i=1}^{N} \operatorname{Fix}\left(T_{i}\right)$. This completes the proof.

For the class of mappings which are asymptotically pseudocontractive, we have the following result immediately.

Corollary 4.2. Let $\mathrm{C}$ be a nonempty closed convex subset of a Hilbert space $\mathrm{H}$ and let $\mathrm{Proj}_{\mathrm{C}}^{\mathrm{H}}$ be the metric projection from $\mathrm{H}$ onto $\mathrm{C}$. Let $\mathrm{T}_{i}: \mathrm{C} \rightarrow \mathrm{E}$ be a uniformly $\mathrm{L}_{i}$-Lipschitz continuous and asymptotically pseudocontractive mapping with the sequence $\left\{k_{n, i}\right\} \subset[1, \infty)$ such that $\sum_{n=1}^{\infty}\left(k_{n, i}-1\right)<\infty$ for each $1 \leqslant i \leqslant N$, where $N \geqslant 1$ is some positive integer. Assume that the common fixed point set $\cap_{i=1}^{N} \operatorname{Fix}\left(\mathrm{T}_{i}\right)$ is nonempty. Let $\left\{x_{n}\right\}_{\mathfrak{n}=0}^{\infty}$ be a sequence generated in the following manner:

$$
x_{n}=\alpha_{n} x_{n-1}+\left(1-\alpha_{n}\right)\left(\operatorname{Proj}_{C}^{H} T_{i(n)}\right)^{h(n)} x_{n}, \quad \forall n \geqslant 1 .
$$

Assume that the control sequence $\left\{\alpha_{n}\right\}$ in $(0,1)$ satisfies the following restrictions $0<1-\frac{1}{\mathrm{~L}}<\mathrm{a} \leqslant \alpha_{n} \leqslant \mathrm{~b}<1$, where

$$
\mathrm{L}=\max \left\{\mathrm{L}_{\mathrm{i}}: 1 \leqslant \mathrm{i} \leqslant \mathrm{N}\right\}, \quad \forall \mathrm{n} \geqslant 1 .
$$

Then $\left\{x_{n}\right\}$ converges weakly to some point in $\cap_{i=1}^{N} \operatorname{Fix}\left(T_{i}\right)$.

\section{Strong convergence theorems}

Recall that a family $\left\{\mathrm{T}_{i}\right\}_{i=1}^{\mathrm{N}}: \mathrm{C} \rightarrow \mathrm{H}$ with $\cap_{i=1}^{\mathrm{N}} \operatorname{Fix}\left(\mathrm{T}_{i}\right) \neq \emptyset$ is said to satisfy Condition (YP) on $\mathrm{C}$ if there is a nondecreasing function $f:[0, \infty) \rightarrow[0, \infty)$ with $f(0)=0$ and $f(m)>0$ for all $m \in(0, \infty)$ such that for all $x \in \mathrm{C}$

$$
\max _{1 \leqslant i \leqslant N}\left\{\left\|x-\left(\operatorname{Proj}_{C}^{H} T_{i}\right) x\right\|\right\} \geqslant f\left(d\left(x, \cap \cap_{i=1}^{N} \operatorname{Fix}\left(T_{i}\right)\right)\right) .
$$

Next, we give strong convergence theorems with the help of Condition (YP).

Theorem 5.1. Let $\mathrm{C}$ be a nonempty closed convex subset of a Hilbert space $\mathrm{H}$ and let $\mathrm{Proj}_{\mathrm{C}}^{\mathrm{H}}$ be the metric projection from $\mathrm{H}$ onto $\mathrm{C}$. Let $\mathrm{T}_{\mathrm{i}}: \mathrm{C} \rightarrow \mathrm{E}$ be a uniformly $\mathrm{L}_{\mathrm{i}}$-Lipschitz continuous and asymptotically pseudocontractive 
mapping in the intermediate sense with the sequence $\left\{k_{n, i}\right\} \subset[1, \infty)$ such that $\sum_{n=1}^{\infty}\left(k_{n, i}-1\right)<\infty$ for each $1 \leqslant i \leqslant N$, where $N \geqslant 1$ is some positive integer. Let

$$
\xi_{(n, i)}=\max \left\{0, \sup _{x, y \in C}\left(\left\|T_{i}^{n} x-T_{i}^{n} y\right\|^{2}-k_{(n, i)}\|x-y\|^{2}-\left\|\left(I-T_{i}^{n}\right) x-\left(I-T_{i}^{n}\right) y\right\|^{2}\right)\right\},
$$

for each $1 \leqslant i \leqslant N$. Assume that the common fixed point set $\cap_{i=1}^{N} \operatorname{Fix}\left(T_{i}\right)$ is nonempty. Let $\left\{x_{n}\right\}_{\mathfrak{n}=0}^{\infty}$ be a sequence generated in the following manner:

$$
x_{n}=\alpha_{n} x_{n-1}+\left(1-\alpha_{n}\right)\left(\operatorname{Proj}_{C}^{H} T_{i(n)}\right)^{h(n)} x_{n}, \quad \forall n \geqslant 1 .
$$

Assume that the control sequence $\left\{\alpha_{n}\right\}$ in $[0,1]$ satisfies the following restrictions $0<1-\frac{1}{\mathrm{~L}}<\mathrm{a} \leqslant \alpha_{n} \leqslant \mathrm{~b}<1$, where

$$
\mathrm{L}=\max \left\{\mathrm{L}_{\mathrm{i}}: 1 \leqslant \mathrm{i} \leqslant \mathrm{N}\right\}, \quad \forall \mathrm{n} \geqslant 1,
$$

$\sum_{n=1}^{\infty} \xi_{n}<\infty$, where $\xi_{n}=\max \left\{\xi_{(n, i)}: 1 \leqslant i \leqslant N\right\}$. If $\left\{T_{1}, T_{2}, \ldots, T_{N}\right\}$ satisfies Condition $(Y P)$, then $\left\{x_{n}\right\}$ converges strongly to some point in $\cap_{i=1}^{\mathrm{N}} \mathrm{Fix}\left(\mathrm{T}_{i}\right)$.

Proof. In view of Condition (YP), using (4.9), we have $\lim _{n \rightarrow \infty} f\left(d\left(x_{n}, \cap_{i=1}^{N} F i x\left(T_{i}\right)\right)\right)=0$, which in turn implies $\lim _{n \rightarrow \infty} d\left(x_{n}, \cap_{i=1}^{N} F i x\left(T_{i}\right)\right)=0$. Now, we are in a position to prove that $\left\{x_{n}\right\}$ is a Cauchy sequence. For any positive integers $m, n$, where $m>n>n_{0}$, we find that

$$
\left\|x_{m}-p\right\| \leqslant Q\left\|x_{n}-p\right\|+Q \sum_{i=n+1}^{\infty} \frac{\xi_{i}}{1-M}+\frac{\xi_{m}}{1-M^{\prime}}
$$

where $Q=\exp \left\{\sum_{n=1}^{\infty} \frac{k_{h(n)}-1}{1-M}\right\}$. Hence, we have

$$
\left\|x_{n}-x_{m}\right\| \leqslant\left\|x_{n}-p\right\|+\left\|x_{m}-p\right\| \leqslant(1+Q)\left\|x_{n}-p\right\|+Q \sum_{i=n+1}^{\infty} \frac{\xi_{i}}{1-M}+\frac{\xi_{m}}{1-M} .
$$

This proves that $\left\{x_{n}\right\}$ is a Cauchy sequence in $C$ and so $\left\{x_{n}\right\}$ converges strongly to some $\bar{q} \in C$. Since each $T_{i}$ is Lipschitz continuous, we see that $\cap_{i=1}^{N} F i x\left(T_{i}\right)$ is a closed set. This in turn implies that $\bar{q} \in \cap_{i=1}^{N} F i x\left(T_{i}\right)$. This completes the proof.

For the class of mappings which are asymptotically pseudocontractive, we have the following result.

Corollary 5.2. Let $\mathrm{C}$ be a nonempty closed convex subset of a Hilbert space $\mathrm{H}$ and let $\mathrm{Proj}_{\mathrm{C}}^{\mathrm{H}}$ be the metric projection from $\mathrm{H}$ onto $\mathrm{C}$. Let $\mathrm{T}_{\mathrm{i}}: \mathrm{C} \rightarrow \mathrm{H}$ be a uniformly $\mathrm{L}_{\mathrm{i}}$-Lipschitz continuous and asymptotically pseudocontractive mapping with the sequence $\left\{k_{n, i}\right\} \subset[1, \infty)$ such that $\sum_{n=1}^{\infty}\left(k_{n, i}-1\right)<\infty$ for each $1 \leqslant i \leqslant N$, where $N \geqslant 1$ is some positive integer. Assume that the common fixed point set $\cap_{i=1}^{N} \operatorname{Fix}\left(T_{i}\right)$ is nonempty. Let $\left\{x_{n}\right\}_{n=0}^{\infty}$ be a sequence generated in the following manner:

$$
x_{n}=\alpha_{n} x_{n-1}+\left(1-\alpha_{n}\right)\left(\operatorname{Proj}_{C}^{H} T_{i(n)}\right)^{h(n)} x_{n}, \quad \forall n \geqslant 1 .
$$

Assume that the control sequence $\left\{\alpha_{n}\right\}$ in $[0,1]$ satisfies the following restrictions $0<1-\frac{1}{\mathrm{~L}}<\mathrm{a} \leqslant \alpha_{\mathrm{n}} \leqslant \mathrm{b}<1$, where $L=\max \left\{L_{i}: 1 \leqslant i \leqslant N\right\}, \forall n \geqslant 1$. If $\left\{T_{1}, T_{2}, \ldots, T_{N}\right\}$ satisfies Condition $(Y P)$, then $\left\{x_{n}\right\}$ converges strongly

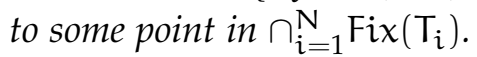

Next, we give another strong convergence theorem with the aid of compactness.

Theorem 5.3. Let $\mathrm{C}$ be a nonempty closed convex subset of a Hilbert space $\mathrm{H}$ and let $\mathrm{Proj}_{\mathrm{C}}^{\mathrm{H}}$ be the metric projection from $\mathrm{H}$ onto $\mathrm{C}$. Let $\mathrm{T}_{\mathrm{i}}: \mathrm{C} \rightarrow \mathrm{H}$ be a uniformly $\mathrm{L}_{\mathrm{i}}$-Lipschitz continuous and asymptotically pseudocontractive mapping in the intermediate sense with the sequence $\left\{k_{n, i}\right\} \subset[1, \infty)$ such that $\sum_{n=1}^{\infty}\left(k_{n, i}-1\right)<\infty$ for each $1 \leqslant i \leqslant N$, where $N \geqslant 1$ is some positive integer. Let

$$
\xi_{(n, i)}=\max \left\{0, \sup _{x, y \in C}\left(\left\|T_{i}^{n} x-T_{i}^{n} y\right\|^{2}-k_{(n, i)}\|x-y\|^{2}-\left\|\left(I-T_{i}^{n}\right) x-\left(I-T_{i}^{n}\right) y\right\|^{2}\right)\right\},
$$

for each $1 \leqslant i \leqslant N$. Assume that the common fixed point set $\cap_{i=1}^{N} \operatorname{Fix}\left(T_{i}\right)$ is nonempty. Let $\left\{x_{n}\right\}_{\mathfrak{n}=0}^{\infty}$ be a sequence 
generated in the following manner:

$$
x_{n}=\alpha_{n} x_{n-1}+\left(1-\alpha_{n}\right)\left(\operatorname{Proj}_{C}^{H} T_{i(n)}\right)^{h(n)} x_{n}, \quad \forall n \geqslant 1 .
$$

Assume that $\left\{\alpha_{n}\right\}$ in $[0,1]$ satisfies the following restrictions $0<1-\frac{1}{L}<a \leqslant \alpha_{n} \leqslant b<1$, where

$$
\mathrm{L}=\max \left\{\mathrm{L}_{\mathrm{i}}: 1 \leqslant \mathrm{i} \leqslant \mathrm{N}\right\}, \quad \forall \mathrm{n} \geqslant 1,
$$

$\sum_{n=1}^{\infty} \xi_{n}<\infty$, where $\xi_{n}=\max \left\{\xi_{(n, i)}: 1 \leqslant i \leqslant N\right\}$. If one of $\left\{T_{1}, T_{2}, \ldots, T_{N}\right\}$ is semicompact, then $\left\{x_{n}\right\}$ converges strongly to some point in $\cap_{i=1}^{N} \mathrm{Fix}\left(\mathrm{T}_{i}\right)$.

Proof. Without loss of generality, we assume that $T_{1}$ is a semicompact mapping. From (4.9), we see that there exits a subsequence $\left\{x_{n_{i}}\right\}$ of $\left\{x_{n}\right\}$ such that it converges strongly to $x \in C$. For each $r \in\{1,2, \ldots, N\}$, we get that

$$
\left\|x-\left(\operatorname{Proj}_{C}^{H} \mathrm{~T}_{\mathrm{r}}\right) x\right\| \leqslant\left\|x_{n_{i}}-\left(\operatorname{Proj}_{C}^{\mathrm{H}} \mathrm{T}_{r}\right) x_{n_{i}}\right\|+\left\|\left(\operatorname{Proj}_{C}^{\mathrm{H}} \mathrm{T}_{\mathrm{r}}\right) x_{n_{i}}-\left(\operatorname{Proj}_{C}^{\mathrm{H}} \mathrm{T}_{\mathrm{r}}\right) x\right\|+\left\|x-x_{n_{i}}\right\| .
$$

Since $\operatorname{Proj}_{C}^{\mathrm{H}}$ and $T_{r}$ are Lipschitz continuous, we obtain that $x \in \cap_{r=1}^{N} F i x\left(\operatorname{Proj}_{C}^{H} T_{r}\right)=\cap_{i=1}^{N} F i x\left(T_{i}\right)$. In view of Theorem 3.1, we obtain that $\lim _{n \rightarrow \infty}\left\|x_{n}-x\right\|$ exists. Therefore, we obtain the desired conclusion immediately. This completes the proof.

For the class of mappings which are asymptotically pseudocontractive, we have the following result.

Corollary 5.4. Let $\mathrm{C}$ be a nonempty closed convex subset of a Hilbert space $\mathrm{H}$ and let $\mathrm{Proj}_{\mathrm{C}}^{\mathrm{H}}$ be the metric projection from $\mathrm{H}$ onto $\mathrm{C}$. Let $\mathrm{T}_{\mathrm{i}}: \mathrm{C} \rightarrow \mathrm{H}$ be a uniformly $\mathrm{L}_{\mathrm{i}}$-Lipschitz continuous and asymptotically pseudocontractive mapping with the sequence $\left\{k_{n, i}\right\} \subset[1, \infty)$ such that $\sum_{n=1}^{\infty}\left(k_{n, i}-1\right)<\infty$ for each $1 \leqslant i \leqslant N$, where $N \geqslant 1$ is some positive integer. Assume that the common fixed point set $\cap_{i=1}^{N} \operatorname{Fix}\left(\mathrm{T}_{i}\right)$ is nonempty. Let $\left\{x_{\mathfrak{n}}\right\}_{\mathfrak{n}=0}^{\infty}$ be a sequence generated in the following manner:

$$
x_{n}=\alpha_{n} x_{n-1}+\left(1-\alpha_{n}\right)\left(\operatorname{Proj}_{C}^{H} T_{i(n)}\right)^{h(n)} x_{n}, \quad \forall n \geqslant 1 .
$$

Assume that $\left\{\alpha_{n}\right\}$ in $[0,1]$ satisfies the following restrictions $0<1-\frac{1}{L}<a \leqslant \alpha_{n} \leqslant b<1$, where

$$
\mathrm{L}=\max \left\{\mathrm{L}_{\mathrm{i}}: 1 \leqslant \mathrm{i} \leqslant \mathrm{N}\right\}, \quad \forall \mathrm{n} \geqslant 1 .
$$

If one of $\left\{\mathrm{T}_{1}, \mathrm{~T}_{2}, \ldots, \mathrm{T}_{\mathrm{N}}\right\}$ is semicompact, then $\left\{\mathrm{x}_{\mathrm{n}}\right\}$ converges strongly to some point in $\cap_{i=1}^{\mathrm{N}} \mathrm{Fix}\left(\mathrm{T}_{i}\right)$.

Finally, we give the following strong convergence criteria.

Theorem 5.5. Let $\mathrm{C}$ be a nonempty closed convex subset of a Hilbert space $\mathrm{H}$ and let Proj $\mathrm{C}_{\mathrm{C}}^{\mathrm{H}}$ be the metric projection from $\mathrm{H}$ onto $\mathrm{C}$. Let $\mathrm{T}_{i}: \mathrm{C} \rightarrow \mathrm{H}$ be a uniformly $\mathrm{L}_{i}$-Lipschitz continuous and asymptotically pseudocontractive mapping in the intermediate sense with the sequence $\left\{k_{n, i}\right\} \subset[1, \infty)$ such that $\sum_{n=1}^{\infty}\left(k_{n, i}-1\right)<\infty$ for each $1 \leqslant i \leqslant N$, where $N \geqslant 1$ is some positive integer. Let

$$
\xi_{(n, i)}=\max \left\{0, \sup _{x, y \in C}\left(\left\|T_{i}^{n} x-T_{i}^{n} y\right\|^{2}-k_{(n, i)}\|x-y\|^{2}-\left\|\left(I-T_{i}^{n}\right) x-\left(I-T_{i}^{n}\right) y\right\|^{2}\right)\right\},
$$

for each $1 \leqslant i \leqslant N$. Assume that $\cap_{i=1}^{N} F i x\left(T_{i}\right)$ is nonempty. Let $\left\{x_{n}\right\}_{\mathfrak{n}=0}^{\infty}$ be a sequence generated in the following manner:

$$
x_{n}=\alpha_{n} x_{n-1}+\left(1-\alpha_{n}\right)\left(\operatorname{Proj}_{C}^{H} T_{i(n)}\right)^{h(n)} x_{n}, \quad \forall n \geqslant 1 .
$$

Assume that $\left\{\alpha_{n}\right\}$ in $[0,1]$ satisfies $0<1-\frac{1}{L}<a \leqslant \alpha_{n} \leqslant b<1$, where

$$
\mathrm{L}=\max \left\{\mathrm{L}_{\mathrm{i}}: 1 \leqslant \mathrm{i} \leqslant \mathrm{N}\right\}, \quad \forall \mathrm{n} \geqslant 1,
$$

$\sum_{n=1}^{\infty} \xi_{n}<\infty$, where $\xi_{n}=\max \left\{\xi_{(n, i)}: 1 \leqslant i \leqslant N\right\}$. Then the sequence $\left\{x_{n}\right\}$ converges strongly to some point in $\cap_{i=1}^{N} \operatorname{Fix}\left(T_{i}\right)$, if and only if $\liminf _{n \rightarrow \infty} d\left(x_{n}, \cap_{i=1}^{N} \operatorname{Fix}\left(T_{i}\right)\right)=0$. 
Proof. The necessity is obvious. We only show the sufficiency. Assume that

$$
\liminf _{n \rightarrow \infty} d\left(x_{n}, \cap_{i=1}^{N} \operatorname{Fix}\left(T_{i}\right)\right)=0 .
$$

In view of Lemma 2.1, we can obtain that $\lim _{n \rightarrow \infty} d\left(x_{n}, \cap_{i=1}^{N} F i x\left(T_{i}\right)\right)=0$. The desired results can be obtained from Theorem 5.1 immediately. This completes the proof.

For the class of mappings which are asymptotically pseudocontractive, we have the following result.

Corollary 5.6. Let $\mathrm{C}$ be a nonempty closed convex subset of a Hilbert space $\mathrm{H}$ and let $\mathrm{Proj}_{\mathrm{C}}^{\mathrm{H}}$ be the metric projection from $\mathrm{H}$ onto $\mathrm{C}$. Let $\mathrm{T}_{\mathrm{i}}: \mathrm{C} \rightarrow \mathrm{H}$ be a uniformly $\mathrm{L}_{i}$-Lipschitz continuous and asymptotically pseudocontractive mapping with the sequence $\left\{k_{n, i}\right\} \subset[1, \infty)$ such that $\sum_{n=1}^{\infty}\left(k_{n, i}-1\right)<\infty$ for each $1 \leqslant i \leqslant N$, where $N \geqslant 1$ is some positive integer. Assume that $\cap_{i=1}^{N}$ Fix $\left(\mathrm{T}_{i}\right)$ is nonempty. Let $\left\{x_{n}\right\}_{\mathfrak{n}=0}^{\infty}$ be a sequence generated in the following manner:

$$
x_{n}=\alpha_{n} x_{n-1}+\left(1-\alpha_{n}\right)\left(\operatorname{Proj}_{C}^{H} T_{i(n)}\right)^{h(n)} x_{n}, \quad \forall n \geqslant 1 .
$$

Assume that $\left\{\alpha_{n}\right\}$ in $[0,1]$ satisfies $0<1-\frac{1}{\mathrm{~L}}<\mathrm{a} \leqslant \alpha_{\mathrm{n}} \leqslant \mathrm{b}<1$, where

$$
\mathrm{L}=\max \left\{\mathrm{L}_{\mathrm{i}}: 1 \leqslant \mathrm{i} \leqslant \mathrm{N}\right\}, \quad \forall \mathrm{n} \geqslant 1 .
$$

Then the sequence $\left\{x_{n}\right\}$ converges strongly to some point in $\cap_{i=1}^{N} \operatorname{Fix}\left(T_{i}\right)$, if and only if

$$
\liminf _{n \rightarrow \infty} d\left(x_{n}, \cap_{i=1}^{N} \operatorname{Fix}\left(T_{i}\right)\right)=0 .
$$

\section{Acknowledgment}

The author is grateful to the referees for useful suggestions which improved the contents of this article.

\section{References}

[1] I. K. Argyros, S. George, On the convergence of inexact Gauss-Newton method for solving singular equations, J. Nonlinear Funct. Anal., 2016 (2016), 22 pages. 1

[2] B. A. Bin Dehaish, A. Latif, H. O. Bakodah, X.-L. Qin, A regularization projection algorithm for various problems with nonlinear mappings in Hilbert spaces, J. Inequal. Appl., 2015 (2015), 14 pages. 1

[3] B. A. Bin Dehaish, X.-L. Qin, A. Latif, H. O. Bakodah, Weak and strong convergence of algorithms for the sum of two accretive operators with applications, J. Nonlinear Convex Anal., 16 (2015), 1321-1336. 1

[4] F. E. Browder, W. V. Petryshyn, Construction of fixed points of nonlinear mappings in Hilbert space, J. Math. Anal. Appl., 20 (1967), 197-228. 2

[5] R. Bruck, T. Kuczumow, S. Reich, Convergence of iterates of asymptotically nonexpansive mappings in Banach spaces with the uniform Opial property, Colloq. Math., 65 (1993), 169-179. 2

[6] S. Y. Cho, B. A. Bin Dehaish, X.-L. Qin, Weak convergence of a splitting algorithm in Hilbert spaces, J. Appl. Anal. Comput., 7 (2017), 427-438. 1

[7] S. Y. Cho, W.-L. Li, S. M. Kang, Convergence analysis of an iterative algorithm for monotone operators, J. Inequal. Appl., 2013 (2013), 14 pages. 1

[8] S. Y. Cho, X.-L. Qin, On the strong convergence of an iterative process for asymptotically strict pseudocontractions and equilibrium problems, Appl. Math. Comput., 235 (2014), 430-438. 2

[9] S. H. Khan, I. Yildirim, M. Ozdemir, Convergence of an implicit algorithm for two families of nonexpansive mappings, Comput. Math. Appl., 59 (2010), 3084-3091. 2

[10] J. K. Kim, S. Y. Cho, X.-L. Qin, Some results on generalized equilibrium problems involving strictly pseudocontractive mappings, Acta Math. Sci. Ser. B Engl. Ed., 31 (2011), 2041-2057. 1

[11] J. K. Kim, Y. M. Nam, J. Y. Sim, Convergence theorems of implicit iterative sequences for a finite family of asymptotically quasi-nonxpansive type mappings, Nonlinear Anal., 71 (2009), e2839-e2848. 2

[12] W. A. Kirk, Fixed point theorems for non-Lipschitzian mappings of asymptotically nonexpansive type, Israel J. Math., 17 (1974), 339-346. 2

[13] Q.-H. Liu, Convergence theorems of the sequence of iterates for asymptotically demicontractive and hemicontractive mappings, Nonlinear Anal., 26 (1996), 1835-1842. 2

[14] S.-T. Lv, Implicit algorithms for a demicontinuous semigroup of pseudocontractions, Commun. Optim. Theory, 2017 (2017), 9 pages. 2 
[15] Z. Opial, Weak convergence of the sequence of successive approximations for nonexpansive mappings, Bull. Amer. Math. Soc., 73 (1967), 591-597. 2

[16] M. O. Osilike, Implicit iteration process for common fixed points of a finite family of strictly pseudocontractive maps, J. Math. Anal. Appl., 294 (2004), 73-81. 2

[17] M. O. Osilike, B. G. Akuchu, Common fixed points of a finite family of asymptotically pseudocontractive maps, Fixed Point Theory Appl., 2004 (2004), 81-88. 2

[18] X.-L. Qin, S. Y. Cho, Convergence analysis of a monotone projection algorithm in reflexive Banach spaces, Acta Math. Sci. Ser. B Engl. Ed., 37 (2017), 488-502. 1

[19] X.-L. Qin, S. Y. Cho, L. Wang, A regularization method for treating zero points of the sum of two monotone operators, Fixed Point Theory Appl., 2014 (2014), 10 pages. 1

[20] X.-L. Qin, J.-C. Yao, Weak convergence of a Mann-like algorithm for nonexpansive and accretive operators, J. Inequal. Appl., 2016 (2016), 9 pages. 1

[21] J. Reinermann, Über Fixpunkte kontrahierender Abbildungen und schwach konvergente Toeplitz-Verfahren, (German) Arch. Math. (Basel), 20 (1969), 59-64. 2.2

[22] D. R. Sahu, H.-K. Xu, J.-C. Yao, Asymptotically strict pseudocontractive mappings in the intermediate sense, Nonlinear Anal., 70 (2009), 3502-3511. 2

[23] J. Schu, Iterative construction of fixed points of asymptotically nonexpansive mappings, J. Math. Anal. Appl., 158 (1991), 407-413. 2

[24] T. V. Su, T. V. Dinh, On the existence of solutions of quasi-equilibrium problems (UPQEP), (LPQEP), (UWQEP) and (LWQEP) and related problems, Commun. Optim. Theory, 2016 (2016), 21 pages. 1

[25] K.-K. Tan, H.-K. Xu, Approximating fixed points of nonexpansive mappings by the Ishikawa iteration process, J. Math. Anal. Appl., 178 (1993), 301-308. 2.1

[26] Z.-M. Wang, Implicit hybrid projection algorithms for common fixed points of a demicontinuous semigroup of pseudocontractions in Hilbert spaces, J. Nonlinear Funct. Anal., 2015 (2015), 12 pages. 2

[27] H.-Y. Zhou, Demiclosedness principle with applications for asymptotically pseudo-contractions in Hilbert spaces, Nonlinear Anal., 70 (2009), 3140-3145. 2 